\title{
The effectiveness of discrete hermite wavelet filters technique in digital image watermarking
}

\author{
Areej M. Abduldaim, Asma Abdulelah Abdulrahman, Fouad Shaker Tahir \\ Branch of Mathematics and Computer Applications, Department of Applied Sciences, University of Technology, Baghdad, Iraq
}

\begin{tabular}{|c|c|}
\hline Article Info & ABSTRACT \\
\hline Article history: & In this work, new discrete wavelets were derived Hermite polynomials for \\
\hline Received Mar 30, 2021 & obtained discrete hermite wavelet transformation (DHWT), and their \\
\hline Revised Jan 12, 2022 & r use in image processing is demonstrated by proving the \\
\hline Accepted Jan 21, 2022 & realization of important theorems. Moreover, the role of the new and \\
\hline & the color image is clarified, and a program was created using Matlab \\
\hline Keywords: & $\begin{array}{l}\text { software by creating a subprogram for constructing the new wavelet and } \\
\text { proving its efficiency with an analytic image. The process is repeated using }\end{array}$ \\
\hline Discrete hermite transform & DHWT to analyze the image. The color image has been subjected to various \\
\hline Embedding algorithm & $\begin{array}{l}\text { attacks after which the watermark is retrieved from the image after } \\
\text { comparing it with the proposed algorithm and it has proven its power faster }\end{array}$ \\
\hline Extracting algorithm & and better than the previously suggested methods. The final conclusion \\
\hline Image watermarking & shows that using new wavelets DHWT better peak signal of noise ratio \\
\hline QR decomposition & $\begin{array}{l}\text { (PSNR)s can be obtained and that the proposed algorithm fills in better the } \\
\text { lack of awareness of the watermark and its strength under different attacks. }\end{array}$ \\
\hline
\end{tabular}

This is an open access article under the CC BY-SA license.



\section{Corresponding Author:}

Asma Abdulelah Abdulrahman

Branch of Mathematics and Computer Applications, Department of Applied Sciences

University of Technology

Baghdad, Iraq

Email: 100243@uotechnology.edu.iq

\section{INTRODUCTION}

The hide information is watermarking to ensure product ownership. The procedure is to provide an image so that confidential information is immersed in the host image [1]-[4]. Using attacks such as Gaussian noise, image extension joint photographic experts group (JPEG) compression and rotation, the results obtained by retrieval of the watermark by applying the proposed algorithm, the watermark is recovered [5]. Discret wavelet transform (DWT) and its effective coefficients were used to apply watermark decoding technology because it contains good filters with high [6], [7]. The robustness of the proposed algorithm is relatively better than some of the previously proposed methods. The inserted watermark image is retrieved from the cover image and the quality of the retrieved watermark is compared with the correlation coefficient. [8]. One of the watermark applications is copyright protection at the highest normalized correlation (NC) level by embedding the watermark after extracting the watermark from the original image [9]. Internet media technologies and rapid expansion to preserve video, image and audio clips [10], [11]. The issue to be solved online for digital video clips from illegal copying [12]. Sethy et al. [13] applied the DWT on the image and embedded the matrix of quick response code (QR code) decomposition factorized watermark in the low low (LL) band of the image and different wavelet filters are applied at the time of watermark insertion and extraction. A comparative study has been also performed to judge the suitability of the scheme, in terms of robustness and quality, on different wavelets for watermarking of color images with matrix factorization. The 
results are tested in terms of extracted watermark quality and watermarked image quality [14]. Watermark with video using lefting wavelet transform (LWT) transformations for the gray watermark to be more transmission using information jamming by embedding with video attacks [10], [15]-[18]. The efficiency of the system has been proven to ensure the security of transmission and compared with other methods. Salman and Abd-Almeer [19] wwareness of the watermark is a requirement to achieve durability and security. Reserch from [20], [21] it produces two matrices after factoring low and upper matrix (LU) to be the principal diameter of 1 . Solving this problem, the benefits of which are summarized in authentication data and copyright protection, so that the process is in two stages, is to hide the watermark information and restore it [22]-[24]. The most important method used, which has a major role in this field is the field of discrete wavelet transform applications have increased very quickly in the use of digital images [25].

In this work, new wavelets were used that were derived from Hermit's polynomials, and many theories were proven to prove their suitability for dealing with images for use in this field. A new program and algorithm were built that helps equip the Matlab program with new wavelets to take its role in implementing many applications in the field of images. And in this work, it was used with the watermark and QR technology. Table 1 To test the proposed algorithm, the images used and the watermark is displayed.

Table 1. Suggested algorithm for watermarking and images

\begin{tabular}{llll}
\hline Watermark & Image a & Image $\mathrm{b}$ & Image $\mathrm{c}$ \\
\hline & & & \\
\hline
\end{tabular}

\section{THE METHOD AND RESEARCH}

The goal in this study is to introduce newly discovered wavelet efficiency to be generated from Hermet polynomials and to use the parent function in wavelet derivation DHWT, in addition, to propose a new and fast algorithm to show the role of these new wavelets in dealing with the blind watermark, use of a matrix decomposition method called QR algorithm which boils down to three steps, the first stage is the implementation of Gram-Schmidt to find the orthogonal the basis of the wavelet's vectors space $\mathrm{W}$ with $\mathrm{V}$, to be used in the second stage as columns of composition $Q$ final step solved in $Q^{T} A=Q^{T}(Q R)=I R=R$ and the effect of wavelet analysis of the image colorful. The QR matrix is affected by the LH block of the image under the influence of the new wavelets DHWT so that the PSNRs are obtained with the best results after proposing two new algorithms so that the proposed method is not affected by the watermark under the influence of different attacks, Table 2 shows the decomposition, Lena, with QR Factorization and the logistic map is a second-degree polynomial whose behavior is chaotic for nonlinear dynamic equations so that its mathematical definition depends on the two values $s$ and $\log i_{0}$ as shown in (1).

$$
\mathrm{x}_{\mathrm{i}+1}=\mathrm{sx}_{\mathrm{i}}\left(1-\mathrm{x}_{\mathrm{i}}\right) \text { where } \mathrm{x}_{\mathrm{i}} \in(0,1)
$$

Table 2. The color image decomposed by QR factorizatio

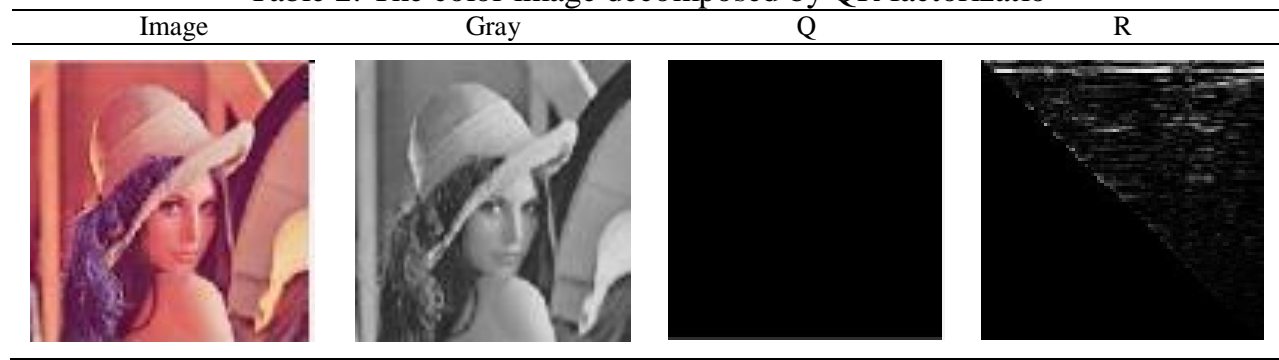




\subsection{Discrete hermite wavelet transform}

The wavelets that are formed from the basic wavelets, by the effect of the factors $(r, s)$ responsible for contraction and expansion:

$$
h_{r, s}(x)=|r|^{\frac{-1}{2}} h\left[\frac{x-s}{r}\right] r, s \in R, r \neq 0
$$

where $h(t)=\left[h_{0}(t), h_{1}(t), \ldots, h_{M-1}\right]^{T}$. The elements $h_{0}(t), h_{1}(t), \ldots, h_{M-1}(t)$ on the $[0,1]$ are orthogonality and basis functions. If $r=2^{-k}, s=(r(2 u-1))$ and transform $x=r\left(2 r^{-1} t\right)$, replacing parameters $\mathrm{r}$, s with a variable in (3) is obtained (2). DHWT $h_{u, v}(t)=h(k, u, v, t)$ includes four parameters, $k \in \mathbb{Z}^{+}, t$ independent variable in $[0,1]$, and $v$ is the degree of the Hermite polynomials:

$$
h_{u, v}(t)=\left\{\begin{array}{l}
2^{\frac{k}{2}} H_{v}^{*}\left(2^{k+1} t-2 u+1\right) t \in\left[\frac{u-1}{2^{k}}, \frac{u}{2^{k}}\right] \\
0 \text { otherwise }
\end{array}\right.
$$

where,

$$
\begin{aligned}
& H_{v}^{*}=\frac{1}{2^{v} v ! \sqrt{\pi}} H_{v} \\
& v=0,1,2, \ldots, M-1 u=0,1,2, \ldots, 2^{k}
\end{aligned}
$$

new wavelets in both vertical and horizontal directions in image analysis and low and high filters are passed on the rows and then on the columns DHWT is applied on a $4 \times 4$ sub-image for more illustration. The coefficients of DHWT obtained above are used where the filter extracted is $(2 \times 2) \mathrm{F}=\frac{1}{\sqrt{\pi}}\left[\begin{array}{cc}1 & -1 \\ 1 & 1\end{array}\right]$ in Figure 1. The analyzed image is divided into four parts that represent the approximation and detail coefficients, namely, LL, HL, LH, and HH.

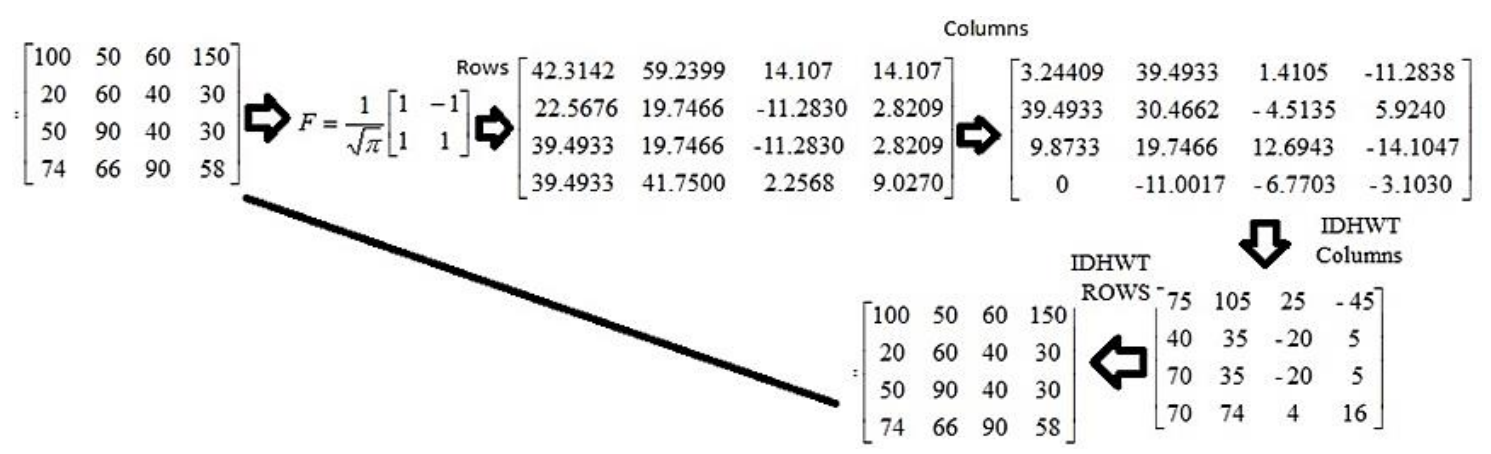

Figure 1. Multi resolution analyses (MRA) with DHWT

\subsection{Watermarking algorithm with DHWT and QR}

In this section, the two proposed algorithms will be implemented using new wavelets DHWT which had a major and important role in image analysis, and the two algorithms are in three stages. The first is in which they are included. Watermark to expose the image with the watermark to several attacks. The embedding scheme using DHWT and QR using logistic map to increase algorithm security by mixing blocks.

\subsection{Watermark embedding algorithm using DHWT and QR}

Steps of embedding with new DHWT:

Step 1: Insert the color image that has a size $M \times M$, and using Matlab, the color image will be converted to grayscale.

Step 2: The image is analyzed using DHWT.

Step 3: The block (LH2) is divided into $2 \times 2$ blocks to be the number of blocks $N \times N$.

Step 4: In this step, the watermark $N \times N$ is inserted, after which the image is converted into binary.

Step 5: The locations are switched for blocks after applying the logistic map as shown in (5). 


$$
i+1=s\left(1-x_{i}\right)
$$

Step 6: Applied algorithm then $A=$ for decomposition to each block.

Step 7: The sub-matrix $\mathrm{R}$ is used to include the binary watermark bits in this matrix from step 6 .

$$
\begin{aligned}
& \mathrm{R}(2,2)=\mathrm{R}(2,2)-\mathrm{R}(2,2) \text { (mod. } \mathrm{r})+T_{1} \text { if } \mathrm{w}=1 \\
& \mathrm{R}(2,2)=\mathrm{R}(2,2)-\mathrm{R}(2,2) \text { (mod. } \mathrm{r})+T_{2} \text { if } \mathrm{w}=0
\end{aligned}
$$

The factor that controls the strength and efficiency of the watermark is $r=20.532$ represented by, $T_{1}=0.75 S, T_{2}=0.25 S$ and Logistics map using $Q R$.

\subsection{Watermark Extracting algorithm using DHWT and QR}

The algorithm shows the detailed watermark extraction steps:

Step 1: The step begins with the watermarked image, which is sized $(M \times M)$ and using the new conversion DHWT convert the image to gray.

Step 2: The second level watermarked image is analyzed using (DHWT).

Step 3: The part (LH2) is chosen, and it is divided into two parts $(2 \times 2)$ to be the number of blocks $(N \times N)$. Step 4: The parts are restored to their original position by applying the chaotic function (logistic map).

Step 5: The matrix $A=Q R$ decomposes all parts of the image.

Step 6: From the R sub-matrix the binary watermark is extracted.

$$
\begin{aligned}
& w=0 \text { if }(\mathrm{T} 1+\mathrm{T} 2) / 2<\bmod (\mathrm{R}(2,2)) \\
& w=1 \text { if }(\mathrm{T} 1+\mathrm{T} 2) / 2 \geq \bmod (\mathrm{R}(2,2))
\end{aligned}
$$

\section{RESULTS AND DISCUSSION}

Through the experiments carried out to evaluate the results, the strength of the proposed watermark algorithm, and the proposed new wavelet effect. The technique and effect of the wavelet DHWT are examined with a watermark on the proposed images using cover images and then converted to grayscale size $512 \times 512$. To be done, divide it into $64 \times 64$ binary chunks as an image watermark. The results were obtained by testing the strength of the proposed algorithm due to the strength of the new wavelets after exposing the watermark image to types of attacks.

Table 3 shows the original used images, the watermark image, and the image with the watermark. Image watermarking technology measures its performance by measuring durability, anonymity, and complexity of calculation. One of the criteria for image quality PSNR is the watermark vision assessment test represented:

$$
\begin{aligned}
& P S N R=10 \log 10\left(\frac{M A X^{2}}{M S E}\right) \\
& M S E=\frac{1}{i j} \sum_{x=0}^{i-1} \sum_{y=0}^{j-1}[I(x, y)-J(x, y)]^{2}
\end{aligned}
$$

The value of the grayscale is equal to 256 , which is the maximum number represented in MAX in equation $\mathrm{W}$ is the watermark, it is calculated a NC between I and I'. The benefit of the new wavelets DHWT is used in two levels on the cover image in the four bands LL2, LH2, HL2 and HH2 to be included in LH2 to be divided into $2 \times 2$ blocks to give 64 blocks and by using the logistic map to increase the safety because it mixes the arrangement QR was used after that, the analysis process begins. The R-matrix in the modulation algorithm using the new waveforms of the second level of the proposed algorithm gave the best results.

$$
N C=\frac{\sum_{x} \sum_{y} W(x, y) W^{\prime}(x, y)}{\sqrt{\sum_{x} W(x, y)} \sqrt{\sum_{x} W^{\prime}(x, y)}}
$$

In Table 3 the results of the proposed algorithm can be seen through the PSNR and NC values for all the watery images. All procedures were tested on the watermark and the robustness of the algorithm was assessed in Table 4 and the effectiveness of the new wavelets DHWT in resisting attacks in Table 5 (see Appendix). As for the comparison in the results obtained in this work are that the $\mathrm{NC}=1$ value obtained is better than that obtained in previous works. 
Table 3. Watermarks in color images with effect DHWT

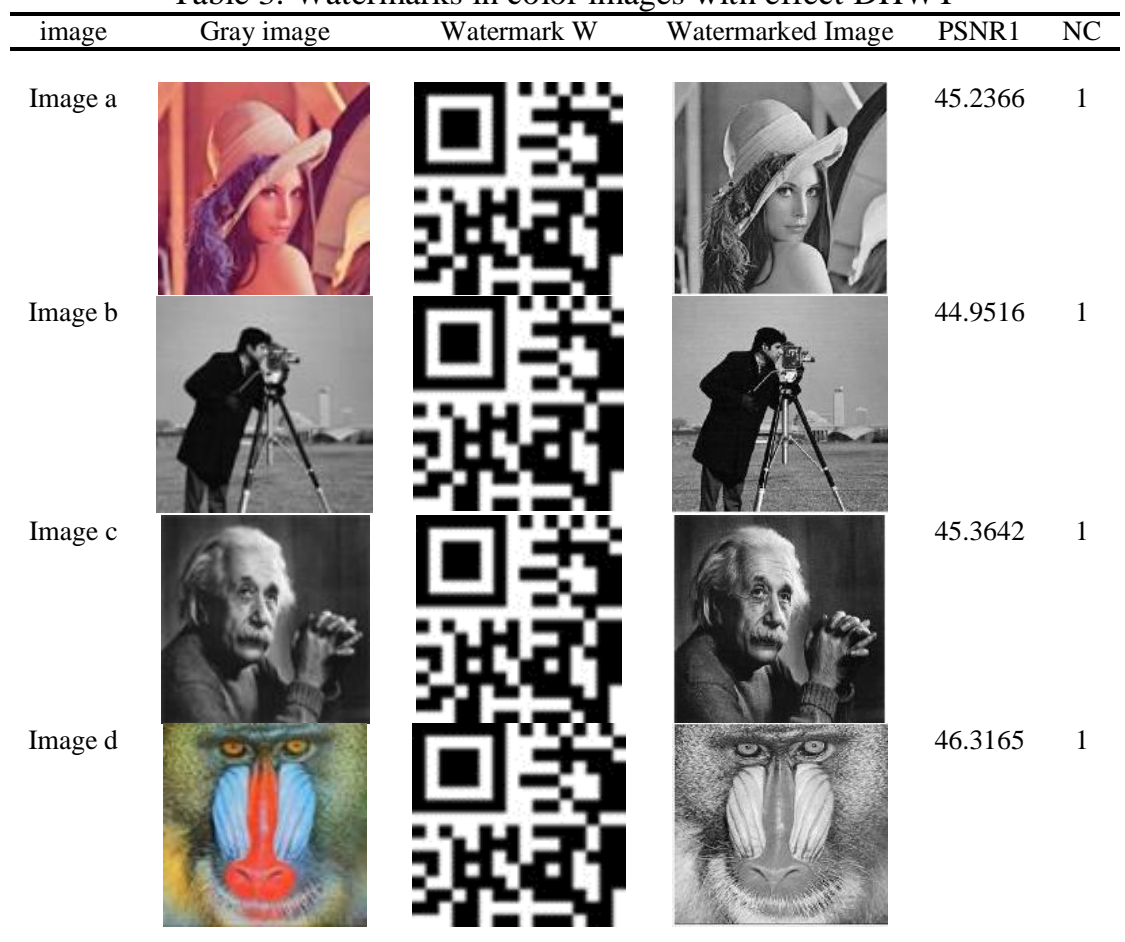

Table 4. Results watermark images with attacks

\begin{tabular}{|c|c|c|c|c|c|c|c|c|c|}
\hline & attacks & $\begin{array}{c}\text { PSNR } \\
\text { image1 }\end{array}$ & $\begin{array}{c}\mathrm{NC} \\
\text { image1 }\end{array}$ & $\begin{array}{l}\text { PSNR } \\
\text { image2 }\end{array}$ & $\begin{array}{c}\mathrm{NC} \\
\text { image2 }\end{array}$ & $\begin{array}{c}\text { PSNR } \\
\text { image3 }\end{array}$ & $\begin{array}{c}\mathrm{NC} \\
\text { image3 }\end{array}$ & $\begin{array}{l}\text { PSNR } \\
\text { image4 }\end{array}$ & $\begin{array}{c}\mathrm{NC} \\
\text { image4 }\end{array}$ \\
\hline 1 & Salt and Pepper \%1 & 27.098 & 0.9999 & 26.6076 & 0.9999 & 26.5350 & 0.9999 & 27.4456 & 0.9999 \\
\hline 2 & Salt and Pepper $\% 5$ & 22.6621 & 0.9999 & 22.3249 & 0.9999 & 22.0151 & 0.9999 & 22.9029 & 0.9999 \\
\hline 3 & JPEGCompression & 59.0931 & 0.9999 & 59.0418 & 0.9999 & 58.8413 & 0.9999 & 58.5980 & 0.9999 \\
\hline 4 & Gaussian noise & 37.6577 & 0.9999 & 37.6711 & 0.9999 & 37.6772 & 0.9999 & 37.6689 & 0.9999 \\
\hline 5 & Histequalization & 19.0572 & 0.9988 & 19.0825 & 0.9988 & 11.9025 & 0.9988 & 16.4756 & 0.9988 \\
\hline 6 & Gaussianlowpassfilter & 33.5516 & 0.9988 & 30.8466 & 0.9988 & 35.3597 & 0.9988 & 30.9082 & 0.9988 \\
\hline 7 & rotate & 33.3455 & 0.9978 & 31.4071 & 0.9978 & 35.3597 & 0.9978 & 30.9082 & 0.9978 \\
\hline 8 & Poisson noise & 27.1281 & 0.9900 & 26.9906 & 0.9900 & 29.4217 & 0.9900 & 27.0017 & 0.9900 \\
\hline 9 & Speckle noise & 35.6501 & 0.9998 & 35.4975 & 0.9998 & 39.0531 & 0.9998 & 35.5068 & 0.9998 \\
\hline 10 & imadjust & 22.1414 & 0.9999 & 19.3848 & 0.9999 & 24.8035 & 0.9999 & 19.3848 & 0.9999 \\
\hline 11 & motionfilter & 25.6241 & 0.9999 & 23.3352 & 0.9999 & 26.1835 & 0.9999 & 23.3352 & 0.9999 \\
\hline 12 & averagefilter & 33.5516 & 0.9999 & 30.9082 & 0.9999 & 35.3598 & 0.9999 & 30.9082 & 0.9999 \\
\hline 13 & medfilter & 31.3918 & 0.9988 & 30.1354 & 0.9988 & 34.4199 & 0.9988 & 30.1335 & 0.9988 \\
\hline 14 & wiener & 38.7865 & 0.9999 & 38.3668 & 0.9999 & 40.3362 & 0.9999 & 34.9624 & 0.9999 \\
\hline 15 & diskfilter & 36.4474 & 0.9988 & 32.2760 & 0.9988 & 36.7007 & 0.9988 & 31.4071 & 0.9988 \\
\hline 16 & ordfilter & 30.0709 & 0.9999 & 36.8095 & 0.9999 & 31.0382 & 0.9999 & 26.8095 & 0.9999 \\
\hline
\end{tabular}

\section{CONCLUSION}

To avoid imitation of the brands of pharmaceutical, industrial and medical products, watermarks with color images were used by the effect of separate waves, and in this work the new wave technology that has proven its efficiency in achieving the best results was used to obtain the best results. The best results. More the quality of the watermark image and its comparison with the standard format waves prepared in Matlab, where this derivation showed the importance and effect of the wavelets used in image processing and analysis with their effective effect in placing the watermark with the color image, the process is repeated using DHWT to analyze the image. The color image was subjected to various attacks, after which the watermark is retrieved from the image after comparing it with the proposed algorithm and it proved its power faster and better than the previously proposed methods. In this work, a new discrete Hermite polynomial wavelet was derived to obtain the discrete hermite wavelet transform (DHWT), and its efficacy for use in image processing has been demonstrated by proving the realization of important theorems. Moreover, the role of the new and proposed waveforms in their effective effect in placing the watermark with the color image was clarified, and a program was created using Matlab software by creating a sub-program for 
constructing the new wave and proving its efficiency through an analytic image. From the final conclusions, PSNR was obtained with high values and $\mathrm{CN}$ close to the ideal value.

\section{APPENDIX}

Table 5. The effectiveness of the new wavelets DHWT in resisting attacks






\section{REFERENCES}

[1] S. L. Galib, F. S. Tahir, and A. A. Abdulrahman, "Detection Face Parts in Image Using Neural Network Based on MATLAB," Engineering and Technology Journal, vol. 39, no. 1B, pp. 159-164, 2021.

[2] N. F. Hassan, A. E. Ali, T. Wala Aldeen, and A. Al-Adhami, "Video mosaic watermarking using plasma key," Indonesian Journal of Electrical Engineering and Computer Science, vol. 22, no. 2, p. 619, May 2021, doi: 10.11591/ijeecs.v22.i2.pp619628 .

[3] S. N. Shihab, A. A. Abdulrahman, and M. N. M. Ali, "Collocation Orthonormal Berntein Polynomials method for Solving Integral Equations," Mathematical Theory and Modeling, vol. 5, no. 12, 2015.

[4] R. K. Singh and D. K. Shaw, "A secured and robust image watermarking based on pixel permutation, wavelet transform and singular value decomposition," Journal of Engineering Science and Technology, vol. 14, no. 4, pp. 1924-1939, 2019.

[5] D. Audichya and V. Soni, "A Review on Colour Image Watermarking based on Wavelet and QR Decomposition," International Research Journal of Engineering and Technology (IRJET), vol. 6, no. 12, pp. 2526-2534, 2019.

[6] C. Li, Y. Yang, K. Liu, and L. Tian, "A Semi-Fragile Video Watermarking Algorithm Based on H.264/AVC," Wireless Communications and Mobile Computing, vol. 2020, pp. 1-11, Jun. 2020, doi: 10.1155/2020/8848553.

[7] C. Wang, R. Shan, and X. Zhou, "Anti-HEVC Recompression Video Watermarking Algorithm Based on the All Phase Biorthogonal Transform and SVD," IETE Technical Review (Institution of Electronics and Telecommunication Engineers, India), vol. 35, no. sup1, pp. 42-58, Dec. 2018, doi: 10.1080/02564602.2018.1477631.

[8] K. U. Singh, V. K. Singh, and A. Singhal, "Color image watermarking scheme based on qr factorization and dwt with compatibility analysis on different wavelet filters," Journal of Advanced Research in Dynamical and Control Systems, vol. 10, no. 6 Special Issue, pp. 1796-1811, 2018.

[9] T. Dutta and H. P. Gupta, "A robust watermarking framework for High Efficiency Video Coding (HEVC) - Encoded video with blind extraction process," Journal of Visual Communication and Image Representation, vol. 38, no. C, pp. 29-44, 2016, doi: 10.1016/j.jvcir.2015.12.007.

[10] H. H. Khairi, S. H. S. Ariffin, N. M. A. Latiff, K. M. Yusof, M. K. Hassan, and M. Rava, "The impact of firewall on TCP and UDP throughput in an openflow software defined network," Indonesian Journal of Electrical Engineering and Computer Science, vol. 20, no. 1, pp. 256-263, Oct. 2020, doi: 10.11591/ijeecs.v20.i1.pp256-263.

[11] H. A. Almurieb and E. S. Bhaya, "Best neural simultaneous approximation," Indonesian Journal of Electrical Engineering and Computer Science, vol. 20, no. 3, pp. 1584-1590, Dec. 2020, doi: 10.11591/ijeecs.v20.i3.pp1584-1590.

[12] B. B. M. Shawkat, A. M. J. Al-Hindawi, and A. H. Shadir, "Spectrum handoff analysis for multiple secondary users in cognitive radio networks," Indonesian Journal of Electrical Engineering and Computer Science, vol. 20, no. 1, pp. 264-274, Oct. 2020, doi: 10.11591/ijeecs.v20.i1.pp264-274.

[13] P. K. Sethy, N. K. Barpanda, A. K. Rath, and S. K. Behera, "Rice false smut detection based on faster R-CNN," Indonesian Journal of Electrical Engineering and Computer Science, vol. 19, no. 3, pp. 1590-1595, Sep. 2020, doi: 10.11591/ijeecs.v19.i3.pp1590-1595.

[14] F. L. Malallah, A. A. Jafaar, N. H. Abbas, and M. G. Saeed, "Reversible color video watermarking scheme based on hybrid of integer-to-integer wavelet transform and Arnold transform," International Journal of Electrical and Computer Engineering, vol. 10, no. 4, pp. 3519-3527, Aug. 2020, doi: 10.11591/ijece.v10i4.pp3519-3527.

[15] S. A. Thajeel, L. M. Kadhim, and S. A. Abdlateef, "A new color image watermarking technique using multiple decompositions," Journal of Theoretical and Applied Information Technology, vol. 95, no. 10, pp. 2323-2331, 2017.

[16] Q. Su, G. Wang, X. Zhang, G. Lv, and B. Chen, "A new algorithm of blind color image watermarking based on LU decomposition," Multidimensional Systems and Signal Processing, vol. 29, no. 3, pp. 1055-1074, Jul. 2018, doi: 10.1007/s11045017-0487-7.

[17] A. M. Abduldaim, “Trivial Extension of $\pi$-Regular Rings,” Engineering and Technology Journal, vol. 34, no. 1, pp. 153-159, 2016

[18] S. A. Salman and A. H. Abd-Almeer, "Clotting detection in the vascular network," Indonesian Journal of Electrical Engineering and Computer Science, vol. 20, no. 2, pp. 1103-1108, Nov. 2020, doi: 10.11591/ijeecs.v20.i2.pp1103-1108.

[19] F. F. Alkhalid, "The effect of optimizers in fingerprint classification model utilizing deep learning," Indonesian Journal of Electrical Engineering and Computer Science, vol. 20, no. 2, pp. 1098-1102, Nov. 2020, doi: 10.11591/ijeecs.v20.i2.pp10981102.

[20] A. A. Abdulrahman and F. S. Tahir, "Face recognition using enhancement discrete wavelet transform based on MATLAB," Indonesian Journal of Electrical Engineering and Computer Science, vol. 23, no. 2, pp. 1128-1136, Aug. 2021, doi: 10.11591/ijeecs.v23.i2.pp1128-1136.

[21] S. L. A. Al-Galib, A. A. Abdulrahman, and F. S. T. Al-Azawi, "Face Detection for Color Image Based on MATLAB," Journal of Physics: Conference Series, vol. 1879, no. 2, p. 022129, May 2021, doi: 10.1088/1742-6596/1879/2/022129.

[22] A. A. Abdulrahman, M. Rasheed, and S. Shihab, "The Analytic of Image Processing Smoothing Spaces Using Wavelet," Journal of Physics: Conference Series, vol. 1879, no. 2, p. 022118, May 2021, doi: 10.1088/1742-6596/1879/2/022118.

[23] S. Shihab, M. Rasheed, O. Alabdali, and A. A. Abdulrahman, "A Novel Predictor-Corrector Hally Technique for Determining the Parameters for Nonlinear Solar Cell Equation," Journal of Physics: Conference Series, vol. 1879, no. 2, p. 022120, May 2021, doi: $10.1088 / 1742-6596 / 1879 / 2 / 022120$.

[24] S. Laith, F. S. Tahir, and A. A. Abdulrahman, "Effectiveness of new algorithms for facial recognition based on deep neural networks," International Journal of Nonlinear Analysis and Applications, vol. 13, no. 1, pp. 2171-2178, 2022, doi: 10.22075/IJNAA.2022.5912.

[25] F. S. Tahir, A. A. Abdulrahman, and Z. H. Thanon, "Novel face detection algorithm with a mask on neural network training," International Journal of Nonlinear Analysis and Applications, vol. 13, no. 1, pp. 209-215, 2022, doi: 10.22075/IJNAA.2022.5472. 


\section{BIOGRAPHIES OF AUTHORS}
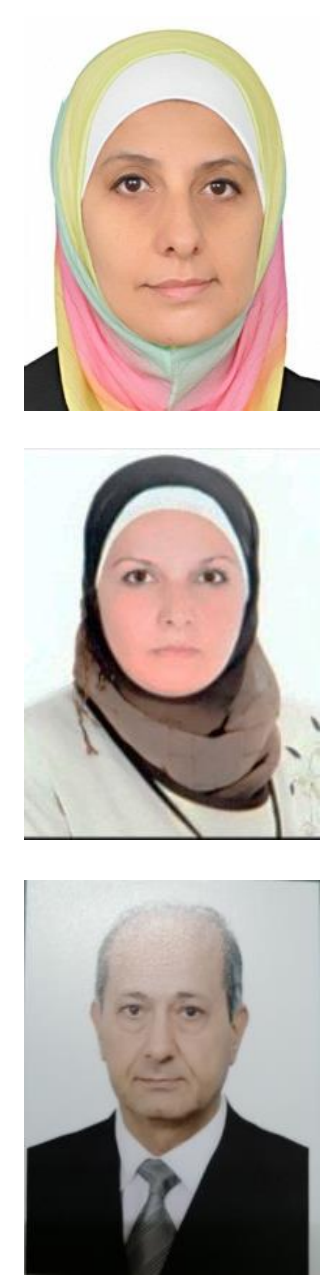

Areej M. Abduldaim (D) S SC P is an Associate Professor at the Branch of Mathematics and Computer Applications, Department of Applied Sciences, University of Technology, Iraq. She received the bachelor's degree (B.Sc.) and the master's degree (M.Sc) in Science of Mathematics from Mustansiriyah University, Iraq in 1995 and 1999 respectively. She received the doctorate degree (Ph.D.) in Fundamental Mathematics from Harbin Institute of Technology, Harbin, China in 2014. Her field of expertise is in the area of Algebra and its Applications and her research interests include Image Processing, Artificial Intelligence, Fuzzy Systems, and Cryptography. She can be contacted at email: 100044@uotechnology.edu.iq.

Asma Abdulelah Abdulrahman (D) SC SC P is an Associate Professor at the Branch of Mathematics and Computer Applications, Department of Applied Sciences, University of Technology, Iraq. She received the bachelor's degree (B.Sc.) 2007and the master's degree (M.Sc) 2012in Science of Mathematics from University of Technology/Baghdad, Ph.D in Image processing/Applied Mathema Acharya Nagarjuna University 2019, Her field of expertise is in the area of Artificial intelligence Applications and her research interests include Image Processing, Artificial Intelligence. She can be contacted at email: 100243@uotechnology.edu.iq.

Fouad Shaker Tahir (iD 8. SC P is an Associate Professor at the Branch of Mathematics and Computer Applications, Department of Applied Sciences, University of Technology, Iraq. He received the bachelor's degree (B.Sc.) and the master's degree (M.Sc) in Science of Mathematics from university of Technology 2004, MSc. Applied Mathematics, University of Technology 1998 BS Electrical Engineering, University of Technology 1984, His field of expertise is in the area of Artificial intelligence Applications and her research interests include Image Processing, Artificial Intelligence, Fuzzy Systems, and Cryptography. He can be contacted at email: 11015@uotechnology.edu.iq. 